\title{
Retrospective case series describing the efficacy, safety and cost-effectiveness of a vial-sharing programme for canakinumab treatment for paediatric patients with cryopyrin-associated periodic syndrome
}

\author{
Abdulkadir A. Elmi ${ }^{1 *}$ D, Karen Wynne², lek L. Cheng ${ }^{2}$, Despina Eleftheriou², Helen J. Lachmann³,
} Philip N. Hawkins ${ }^{3}$ and Paul Brogan ${ }^{1,2}$

\begin{abstract}
Background: Cryopyrin-associated periodic syndrome (CAPS) is a rare autoinflammatory disease, caused by gain of function mutation in NLRP3 resulting in excess production of interleukin-1 (IL-1). Canakinumab is a human monoclonal antibody against Interleukin-1 beta (IL-1 $\beta$ ), licensed for the treatment of CAPS. The objective of the study was to describe the feasibility and cost-effectiveness of a canakinumab vial-sharing programme for paediatric patients with CAPS.

Method: Retrospective case series and clinical service description of a national specially commissioned CAPS clinic at Great Ormond Street Hospital (GOSH). Effectiveness was assessed using a CAPS disease activity score (DAS) and serum amyloid A protein (SAA). Adverse events were collected to determine safety. The number of canakinumab vials saved was considered when investigating the cost-effectiveness of vial-sharing.

Results: Nineteen/20 (95\%) of our paediatric patients achieved minimally active clinical disease activity with canakinumab monotherapy; and 75\% achieved both minimally active clinical disease and serological remission using a pre-specified definition based on the CAPS DAS and SAA level. Canakinumab was well tolerated, with only one child developing an infection requiring hospitalisation during the study. Canakinumab vial sharing resulted in 117 vials of canakinumab saved over a 24-month period, equating to a direct drug-related cost saving of $£ 1,385,821$, and a conservative estimated 5-year cost-saving of $£ 3,464,552.50$.
\end{abstract}

Conclusion: We provide further evidence for the effectiveness and safety of canakinumab in children with CAPS, and highlight the cost-effectiveness of a vial-sharing programme for this high cost medicine. We suggest that this could have important implications for the delivery of other high cost medicines used in paediatric practice.

Keywords: Canakinumab, Cryopyrin-associated periodic syndrome (CAPS), Vial-sharing, Child, Paediatric

\footnotetext{
* Correspondence: zchaael@ucl.ac.uk

'Infection Inflammation and Rheumatology Section, University College

London Institute of Child Health, 30 Guilford St, London WC1N1EH, UK

Full list of author information is available at the end of the article
}

(c) The Author(s). 2019 Open Access This article is distributed under the terms of the Creative Commons Attribution 4.0 International License (http://creativecommons.org/licenses/by/4.0/), which permits unrestricted use, distribution, and reproduction in any medium, provided you give appropriate credit to the original author(s) and the source, provide a link to the Creative Commons license, and indicate if changes were made. The Creative Commons Public Domain Dedication waiver (http://creativecommons.org/publicdomain/zero/1.0/) applies to the data made available in this article, unless otherwise stated. 


\section{Background}

Cryopyrin-associated periodic syndromes (CAPS) are a group of rare, monogenic, autoinflammatory diseases caused by a gain of function mutation in NLR family pyrin domain containing 3 (NLRP3), which results in excess production of interleukin-1 beta (IL-1 $\beta$ ) [1]. This triggers disabling multi-system inflammation from birth, with systemic and organ-specific complications of varying severity, and a long-term risk of reactive, AA amyloidosis. Three main phenotypes are recognised on a continuum of severity [1-3]. The most severe form of CAPS is Chronic Infantile Neurological Cutaneous Articular syndrome (CINCA), associated with severe systemic inflammation from early in life with urticarial rash, central nervous system inflammation, neurological impairment, various inflammatory ophthalmological sequelae, severe musculoskeletal morbidity, and impairment of growth and development. Muckle-Wells syndrome (MWS) is of intermediate severity, with skin rash, periodic fever, and risk of sensorineural hearing loss. The mildest form is familial cold-induced autoinflammatory syndrome (FCAS), typified by cold-induced urticaria, myalgia, malaise and fatigue. There is a surprising lack of genotype-phenotype correlation and little reliable prediction of disease severity based on specific NLRP3 mutations [4].

Canakinumab is a high affinity IgG1 monoclonal antibody against interleukin $1 \beta$ which first received a licence for the treatment of CAPS in 2009. Canakinumab is currently licensed for CAPS in patients from the age of 2 years upwards, and is highly efficacious in this context, resulting in rapid relief of symptoms and normalisation of acute-phase responses in most patients with CAPS [5].

We previously described the paediatric component of a new nationally commissioned highly specialised service for patients in England with CAPS, adding real-world experience regarding the use of canakinumab in a paediatric setting, with outcome data subsequently updated and published online every 2 years [3] (https://www. gosh.nhs.uk/conditions-and-treatments/clinical-outcomes/ rheumatology-clinical-outcomes). Over the first 2 years of this clinic, it became increasingly apparent that dosing young children using adult sized vials (150 mg per vial) resulted in considerable drug wastage, since paediatric doses are based on weight, typically starting at $2 \mathrm{mg} / \mathrm{kg}$ subcutaneously every 8 weeks for children $10 \mathrm{~kg}$ upwards. To address this issue, we introduced a vialsharing programme for children receiving canakinumab for CAPS at Great Ormond Street Hospital NHS Foundation Trust (GOSH). The purpose of this study was to describe the impact of this innovation, and describe generic lessons learned that could have implications when considering similar cost saving initiatives for other high-cost medicines.

\section{Materials and methods}

\section{Aims and objectives}

The aim of the project was to report the clinical and cost-effectiveness of a vial-sharing programme for canakinumab treatment in paediatric patients with CAPS at the Nationally Specialist Commissioned CAPS clinic at Great Ormond Street Hospital (GOSH) NHS Foundation Trust.

The objectives of this project were to:

1. Describe the demographics of the expanding cohort of paediatric patients attending a national specialist clinic for CAPS at GOSH.

2. Describe the therapeutic effectiveness of canakinumab, using a standardised disease activity score and a sensitive serological marker of inflammation (Serum amyloid A).

3. Describe safety based on adverse events.

4. Assess the cost of canakinumab in this context, and the economic impact of a systematic vial-sharing program at GOSH, including the development of a spreadsheet package to facilitate this analysis retrospectively, and to model future cost savings based on projected growth of the clinical service over the next 5 years.

\section{Design and patients}

This was a single-centre, retrospective, observational study of paediatric CAPS patients receiving canakinumab between November 1st 2015, and October 31st 2017 at the national specially commissioned CAPS Clinic at GOSH. This is the only specialised paediatric service across the United Kingdom for patients with this ultra-rare autosomal dominant genetic disease. Since this was a retrospective case notes review with presentation of fully anonymised data, written patient/parental consent was not required. Patients with CAPS were eligible for canakinumab treatment if they were $\geq 2$ years of age; had clinical features of active CAPS requiring medical intervention; and had a confirmed mutation in the NLRP3 gene; or had characteristic clinical features of CAPS if they were NLRP3 mutation negative [6]. Exclusion criteria were evidence of a pre-existing underlying infection or any other significant medical condition that would preclude treatment with canakinumab. Research and ethical approval for this retrospective study was granted from the Joint Research and Development Office of Great Ormond Street Hospital, in accordance with UK law (reference number 16IR47).

\section{Assessment of CAPS disease activity}

A standardised approach was used to monitor CAPS disease activity, as described previously in this clinic [3]. In brief, this consisted of standardised documentation of 
clinical disease activity using the CAPS disease activity score (DAS) (please see Additional file 1). The DAS depicts disease activity using ten symptoms/signs; absence of disease activity was defined as a score of $0 / 20$; minimal disease activity was depicted by a score $\leq 3 / 20$; and a maximum score of 20 indicated maximal disease activity [3]. Serological response was monitored using serum amyloid A (SAA, reference range $<10 \mathrm{mg} / \mathrm{L}$ ). Using these indices, minimally active disease was defined as absent or minimal disease activity (DAS $\leq 3 / 20$ with no item scoring as severe) and normal markers of inflammation (SAA $<10 \mathrm{mg} / \mathrm{L}$ ). The CAPS DAS and SAA (plus other routine investigations: full blood count, renal and liver function; and C-reactive protein) were assessed as a minimum every 2 months from commencement of treatment.

Canakinumab vial sharing and assessment of cost savings Vial sharing was instituted as part of the clinical CAPS service at GOSH in November 2015. The paediatric CAPS clinic occurs monthly, and all patients attend GOSH for clinical assessment, laboratory investigation and dosing. One important advantage of centralisation of care for this very rare disease is that it can facilitate vial sharing of canakinumab: individual patient doses (typically, 10 patients per clinic) are made up centrally in the pharmacy aseptic unit using the required number of vials to generate these doses resulting in minimum wastage.

For a full description of how canakinumab is reconstituted, we refer the reader to the summary of product characteristics, available at: (https://www.ema.europa.eu/ en/documents/product-information/ilaris-epar-productinformation_en.pdf). In brief, canakinumab comes in vials containing $150 \mathrm{mg}$ powder for reconstitution with $1 \mathrm{ml}$ of clear and colourless solvent. After reconstitution, it has a 24 -h refrigerated $\left(4-8^{\circ} \mathrm{C}\right)$ shelf-life. Exact doses for individual patients are taken from these reconstituted vials aseptically in pharmacy, for subcutaneous injection into the patient on the ward on the same day as clinic.

The cost of canakinumab (before and after value added tax, VAT) was derived from the National Institute for Health and Care Excellence [Accessed April 21, 2017] (https://www.nice.org.uk/advice/esnm23/chapter/ key-points-from-the-evidence). From that, direct cost savings were calculated by subtracting the cost of actual number of vials used from the number of vials that would have been required if one (or more, if under exceptional circumstances the canakinumab dose exceeded $150 \mathrm{mg}$ ) vials had been allocated to each patient. Pharmacy costs required to deliver central drug preparation were also taken into account when calculating the final cost saving. Using this approach, the actual cost saving was calculated for a 24 month period starting from
November 1st, 2015 (i.e. spanning 24 clinics). Conservative projected cost savings based on this were extrapolated over the next 5 years.

\section{Data handling and statistics}

The project utilised descriptive statistics for categorical data, and summarised as percentage unless otherwise stated; numeric data were expressed as median and range. The differences in categorical variables were investigated using Fisher's exact test with significance set at a two-tailed $p$-value of less than 0.05 . Fisher's exact test was calculated using a freely available online software (https:// www.graphpad.com/quickcalcs/contingencyl.cfm). Microsoft Excel 2016 was used to produce a spreadsheet used to investigate the cost savings of vial-sharing to produce graphical outputs.

\section{Results}

Baseline clinical features

Since its inception in 2011, the paediatric CAPS service at GOSH has expanded every year, from 14 patients in 2013 to 39 patients who had attended the children's CAPS service by the end of 2016. At the time of this study, the number of patients currently treated by the service was 28 (11 having been successfully transitioned to adult care). Of these, $20 / 28$ patients who were receiving canakinumab were included in this study (Table 1). The remaining eight patients were excluded due to receiving anakinra treatment $(n=3)$ or awaiting treatment to be commenced $(n=5)$.

\section{Response to canakinumab}

Before commencing canakinumab treatment, the median CAPS DAS was $6.5 / 20$ (range $0-11 / 20$ ), with $95 \%$ of patients scoring $>3 / 20$ (Fig. 1). The median SAA before treatment was $34.8 \mathrm{mg} / \mathrm{L}$ (range 0-497; reference range [RR] less than $10 \mathrm{mg} / \mathrm{L}$ ); and SAA was raised (higher

Table 1 Summary of patients attending the national paediatric cryopyrin associated periodic fever syndrome service

\begin{tabular}{|c|c|c|}
\hline \multicolumn{2}{|l|}{$\begin{array}{l}\text { Total number of patients who ever attended the } \\
\text { GOSH CAPS service }\end{array}$} & 39 \\
\hline \multicolumn{2}{|l|}{ Total number of patients included for study } & 20 \\
\hline \multicolumn{2}{|l|}{$\begin{array}{l}\text { Total number of patients with negative } \\
\text { NLRP3 mutation }\end{array}$} & 4 \\
\hline \multirow{2}{*}{$\begin{array}{l}\text { Total number of doses of canakinumab } \\
\text { ever received per patient }\end{array}$} & Median & 14 \\
\hline & Range & $7-41$ doses \\
\hline \multirow[t]{2}{*}{ Age } & Median & 9 years \\
\hline & Range & $4-14$ years \\
\hline \multirow[t]{2}{*}{ Gender } & Male & 10 \\
\hline & Female & 10 \\
\hline
\end{tabular}

CAPS Cryopyrin associated periodic fever syndrome, GOSH Great Ormond Street Hospital 
- $C$ APS DAS $\leq 3 / 20 \quad$ CAPS DAS $>3$

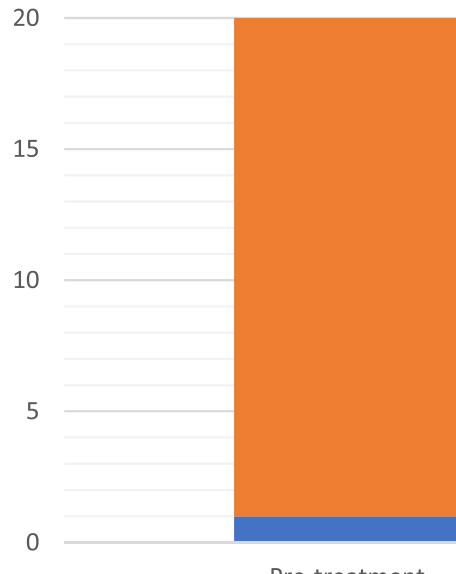

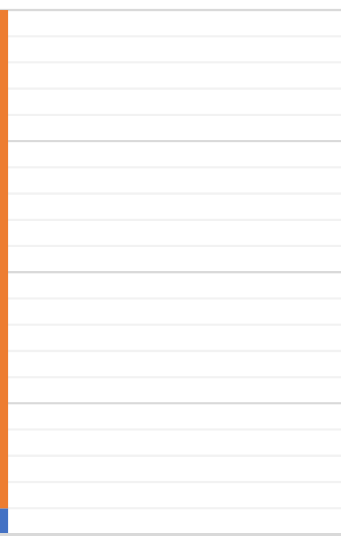

Pre-treatment

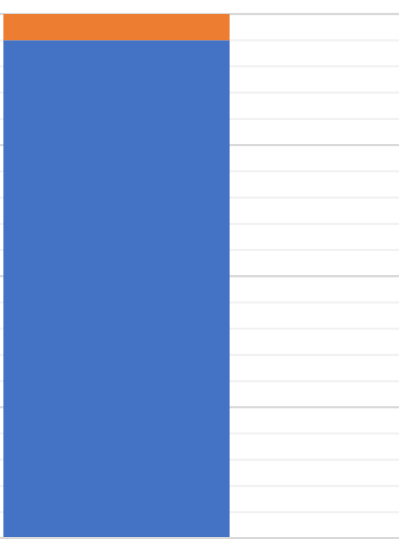

On treatment

Fig. 1 Cryopyrin associated periodic syndrome disease activity score pre-and post canakinumab. The difference between CAPS DAS scores before and after canakinumab treatment was highly significant $(P<0.0001)$. A standardised approach was used to monitor CAPS disease activity, as described previously in this clinic [3]. In brief, this consisted of standardised documentation of clinical disease activity using the CAPS disease activity score (DAS) (please see Additional file 1). The DAS depicts disease activity using ten symptoms/signs; absence of disease activity was defined as a score of 0/20; minimal disease activity was depicted by a score $\leq 3 / 20$; and a maximum score of 20 indicated maximal disease activity. The figure shows that before commencing canakinumab treatment, the median CAPS DAS was 6.5/20 (range 0-11/20), with $95 \%$ of patients scoring $>3 / 20$ (Fig. 1)

than upper RR) in $11 / 20$ (55\%) patients (Fig. 2). On canakinumab, 19/20 (95\%) patients had a CAPS DAS $\leq 3$ (Fig. 1); and 14/20 (70\%) patients had a SAA of $<10 \mathrm{mg} /$ L (Fig. 2). After canakinumab treatment, $15 / 20$ (75\%) patients had CAPS DAS $\leq 3$ and a SAA $<10 \mathrm{mg} / \mathrm{L}$ (Fig. 3).

\section{Safety of canakinumab}

Seven patients developed infections after a median treatment period of 16 months (range 2-33). These were: bacterial tracheitis $(n=1)$; presumed viral upper respiratory tract infection $(n=1)$; lower respiratory tract infection, no organism documented $(n=3)$; uncomplicated varicella zoster virus infection $(n=1)$; presumed viral gastroenteritis $(n=1)$. Only one infection episode resulted in hospitalisation for intravenous antibiotics (the case of bacterial tracheitis, no organism isolated, but prompt response to antibiotic therapy). There were no other adverse effects documented; in particular, there were no injection site reactions reported.

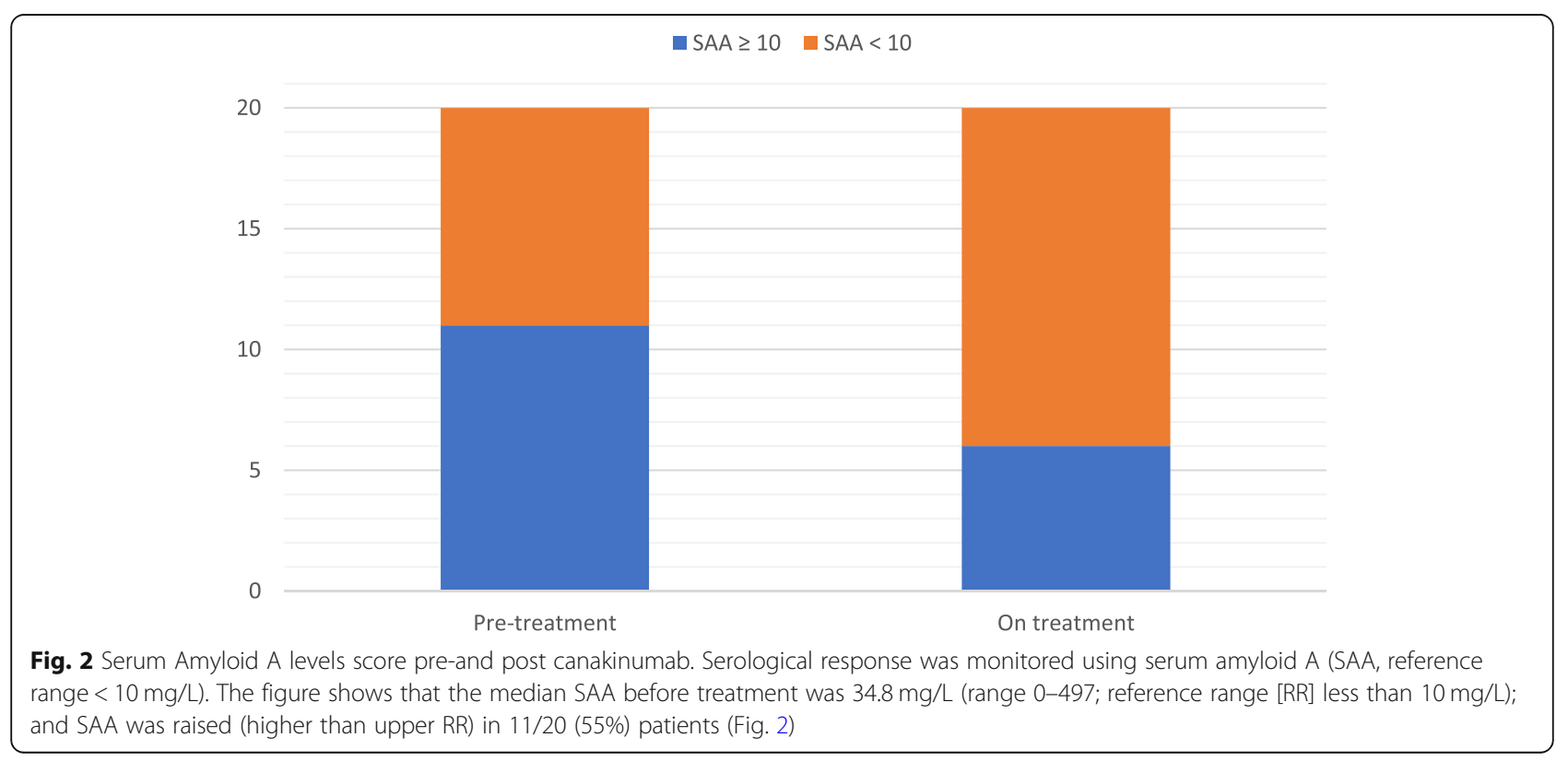


- SAA $\geq 10$ and/or CAPS DAS $>3 \quad$ SAA $<10$ and CAPS DAS $\leq 3$
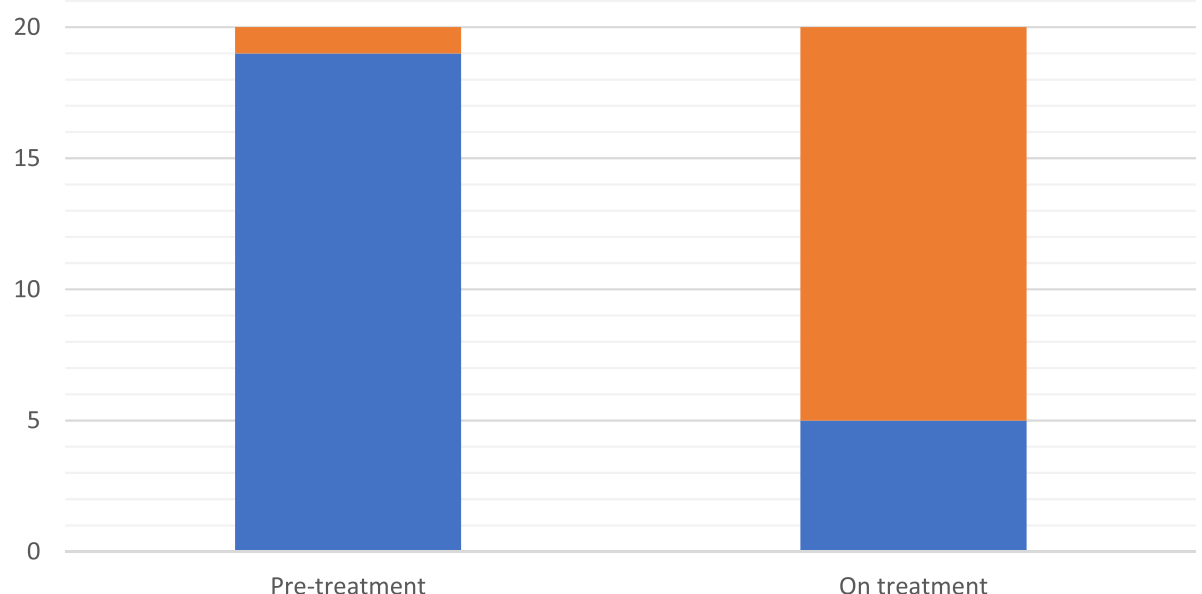

On treatment

Fig. 3 CAPS disease activity score and Serum Amyloid A levels score pre-and post canakinumab. The difference between CAPS DAS and SAA scores before and after canakinumab was significant $(P<0.0001)$. Using these indices, minimally active disease was defined as absent or minimal disease activity (DAS $\leq 3 / 20$ with no item scoring as severe) and normal markers of inflammation (SAA $<10 \mathrm{mg} / \mathrm{L}$ ). The CAPS DAS and SAA (plus other routine investigations: full blood count, renal and liver function; and C-reactive protein) were assessed as a minimum every 2 months from commencement of treatment. On canakinumab, $19 / 20$ (95\%) patients had a CAPS DAS $\leq 3$ (Fig. 1); and 14/20 (70\%) patients had a SAA of < 10 mg/L (Fig. 2). After canakinumab treatment, $15 / 20$ (75\%) patients had CAPS DAS $\leq 3$ and a SAA $<10 \mathrm{mg} / \mathrm{L}$ (Fig. 3)

\section{Savings with Canakinumab vial-sharing}

Table 2 summarises the actual direct drug cost saving over a 24-month period starting from November 1st 2015. The total cost of one vial of canakinumab before value added tax (VAT), as stated by both the National Institute for Health and Care Excellence (NICE) and British National Formulary (BNF), is $£ 9927.80$ (https://www.nice.org.uk/advice/esnm23/chapter/key-pointsfrom-the-evidence). The cost for a single vial after VAT is $£ 11,913$.

Vial sharing resulted in an actual direct drug cost saving of $£ 1,393,821$ over a 24 -month period. Subtracting costs associated with central pharmacy canakinumab preparation ( $£ 4000$ per annum), the net annual cost

Table 2 Actual (2015-2017) and 5-year projected cost savings from canakinumab vial-sharing

\begin{tabular}{|c|c|}
\hline Total number of vials used if no vial sharing ${ }^{a}$ & 268 \\
\hline Total number of vials used with vial sharing ${ }^{a}$ & 151 \\
\hline Total number of vials saved over 24 months $^{a}$ & 117 \\
\hline Cost of one $150 \mathrm{mg}$ canakinumab vial (excluding VAT) & $£ 9927.80$ \\
\hline Cost of one 150 mg canakinumab vial (including VAT) & $£ 11,913$ \\
\hline Total drug cost without vial sharing ${ }^{b}$ & $£ 3,192,684$ \\
\hline Total drug cost with vial sharing ${ }^{b}$ & $£ 1,798,863$ \\
\hline $\begin{array}{l}\text { Cost saving from vial sharing over } 24 \text { months } \\
\text { (Including preparation cost) }\end{array}$ & $£ 1,385,821$ \\
\hline $\begin{array}{l}\text { Projected net cost saving over the next five years }{ }^{c} \\
\text { (Including preparation cost) }\end{array}$ & $£ 3,464,552.50$ \\
\hline
\end{tabular}

a 1.11.15-31.10.17, spanning 24 clinics; ${ }^{\text {b}}$ Total costs includes VAT, since canakinumab administered in hospital; 'assuming no growth in patient numbers, based on net cost saving 2015-2017 saving was $£ 1,385,821$, providing a 5 -year projected net cost saving of approximately $£ 3.5$ million (assuming no further growth in patient numbers).

\section{Discussion}

Controlled and uncontrolled studies unequivocally demonstrate that canakinumab is a highly effective and safe treatment for children and adults with CAPS [3], as reinforced by the data presented here. In the context of a national specially commissioned paediatric CAPS clinical service, $95 \%$ of our paediatric patients achieved minimally active clinical disease activity; and $75 \%$ achieved both minimally active clinical disease and serological remission using a pre-specified definition based on the CAPS DAS and SAA level. Moreover, canakinumab was well tolerated, with only one child developing an infection requiring hospitalisation. Thus, canakinumab is effective and safe in children with CAPS. Canakinumab, however, is expensive costing approximately $£ 12,000$ per vial, and many children require less than one vial per dose. To address this, we designed our clinical service to facilitate vial sharing having observed considerable drug wastage in the first 4 years of this specialist clinic.

We first introduced this vial sharing programme in November 2015, and explored the relative merits but also potential downsides of such an approach over the next 24 months. A total of 117 vials were saved over a 24-month period (24 clinics), providing a cost saving of approximately $£ 1.39$ million (which includes VAT, since in the UK this is added to the cost of medicines administered to patients in hospital), after subtracting the 
modest cost associated with central pharmacy preparation of individual patient doses. To provide a conservative estimate of the projected 5-year cost savings, we assumed that the number of patients treated and number of vials used would remain constant, which provided an estimate of a $£ 3.5 \mathrm{~m}$ saving over 5 years. Given that the paediatric CAPS service has thus far grown yearly since 2011, the actual cost saving may turn out to be considerably higher. However, we acknowledge that this is a crude 5-year estimate that could be affected by changes in patient doses over time that may influence number of vials saved, and other market forces such as changes in the price of canakinumab (although we are not aware of any plans to change the price of canakinumab in the foreseeable future).

This model could have implications for the use of other high-cost children's medicines. Our simple innovation was entirely dependent on running the clinic on the same day of the month for all CAPS patients, which enabled us to a priori calculate the total clinic dose required 2 weeks in advance. Centralised pharmacy preparation of individual doses used a much reduced number of canakinumab vials and only required very modest investment ( $£ 4000$ per annum). We suggest that this innovation will help secure resilience for paediatric CAPS patient access to this high cost medicine for years to come, whilst generating considerable savings for the NHS for the foreseeable future. It also improves patient safety by reducing the likelihood of medication errors since drug is reconstituted aseptically centrally by a dedicated hospital pharmacist. Furthermore, it improves the patient experience by reducing their length of visit as doses are prepared ahead of their visits.
General points to consider when contemplating vialsharing for other high cost medicines are summarised in Table 3. Whilst there are clear benefits of centralisation of highly specialist clinical services, such as (importantly) accrual of unique clinical expertise as a direct consequence of high throughput of patients with an exceptionally rare disease; and the highlighted economic benefits we describe here in; two important downsides are noteworthy. First and foremost, patients have to travel, sometimes from afar, to the centre of expertise. This is on the whole manageable for patients with CAPS, since dosing is every 8 weeks, and additionally centralises and consolidates clinical expertise for this ultra-rare disease. Secondly, it is not possible to make real-time dose changes on the day of the clinic since the canakinumab is prescribed and prepared in advance. In practice this is not a major issue for CAPS patients, because canakinumab has a very long half-life (approximately 28 days), and therefore dose alterations can safely occur at the next clinic visit (Table 3). These latter 2 points are however important to consider when contemplating vial sharing for other high cost medicines.

\section{Conclusion}

In conclusion, canakinumab is highly effective for paediatric patients with CAPS, and demonstrates excellent safety and tolerability. We demonstrate the costeffectiveness of a canakinumab vial-sharing programme, which inarguably offsets some of the high cost associated with this treatment. We suggest that this approach could be applied to other high-cost medicines used in paediatric practice.

Table 3 Suggested characteristics of a successful vial sharing programme for high cost medicines

\begin{tabular}{|c|c|}
\hline Characteristic & Comments \\
\hline Well defined patient population & $\begin{array}{l}\text { Published diagnostic criteria for CAPS are available, and genetic testing for NLRP3 mutations } \\
\text { is available. }\end{array}$ \\
\hline Expensive medicine & The cost of one vial of canakinumab is $£ 11,913$. \\
\hline Risk of drug wastage in paediatric population & $\begin{array}{l}\text { Example: A } 10 \mathrm{~kg} \text { baby with CAPS would have a typical starting dose of } 20 \mathrm{mg} \text {. This means } \\
\text { that without vial sharing } 130 \mathrm{mg} \text { of the vial would be wasted. }\end{array}$ \\
\hline Patients attend single centre on same day as dose & This service only applies to NHS England patients; some travel long distances every 8 weeks. \\
\hline $\begin{array}{l}\text { Dosage interval acceptable for regular travel } \\
\text { to single centre }\end{array}$ & $\begin{array}{l}\text { Patients receive canakinumab every } 8 \text { weeks, minimising impact on families of regular travel } \\
\text { to the national CAPS clinic. }\end{array}$ \\
\hline $\begin{array}{l}\text { Sufficient numbers of patients to make vial } \\
\text { sharing feasible }\end{array}$ & $\begin{array}{l}\text { Whilst CAPS as an exceptionally rare disease, centralisation of the national clinic provides } \\
\text { sufficient patient numbers to ensure that vial sharing becomes a feasible and cost-effective } \\
\text { exercise. }\end{array}$ \\
\hline Availability of a pharmacy aseptic unit & $\begin{array}{l}\text { Individual doses need to be made under aseptic conditions to allow vial sharing. Centres will } \\
\text { require an aseptic unit with the capacity to fulfil the demand to implement this innovative } \\
\text { practice. }\end{array}$ \\
\hline Nationally commissioned service in place & $\begin{array}{l}\text { National funding arrangement agreed from NHS England for the use of canakinumab in } \\
\text { patients with CAPS. This was a key aspect facilitating centralisation of care, and thus a viable } \\
\text { vial sharing programme. }\end{array}$ \\
\hline Acute "real-time" changes in drug dose not critical & $\begin{array}{l}\text { Changes in canakinumab dose cannot be made on the day of clinic since this is prescribed } \\
2 \text { weeks in advance. Dosage alteration therefore occurs } 8 \text { weeks later at the next clinic visit. }\end{array}$ \\
\hline
\end{tabular}




\section{Additional file}

Additional file 1: Canakinumab clinical assessment pro forma. (DOCX 63 kb)

\begin{abstract}
Abbreviations
BNF: British National Formulary; CAPS: Cryopyrin-associated periodic syndrome; CINCA: Chronic infantile cutaneous neurological articular syndrome; DAS: Disease activity score; FCAS: Familial cold-induced autoinflammatory syndrome; GOSH: Great Ormond Street Hospital; IL-1 $\beta$ : Interleukin-1 beta; MWS: Muckle-Wells syndrome; NHS: National Health Service; NICE: National Institute for Health and Care Excellence; NLRP3: NLR family pyrin domain containing 3; R\&D: Research and Development; SAA: Serum Amyloid A
\end{abstract}

\section{Acknowledgments}

We acknowledge the help of Dr. Liban Elmi for proof reading the final draft of the manuscript.

\section{Authors' contributions}

AE helped design the study, collected data and wrote the first draft of the manuscript. KW helped collect the primary data, and contributed to the drafting of the manuscript. ILC helped collect the primary data, particularly in relation to pharmacy issues, and contributed to the drafting of the manuscript. DE was involved in the clinical care of the patients, collected data, and contributed to drafting the manuscript. HL collected data and contributed to drafting the manuscript. PH helped with study conception, collected data and contributed to drafting the manuscript. PAB conceived the study design, and contributed to the first and all subsequent drafts of the manuscript, including approval of the final draft ready for submission. All authors read and approved the final manuscript.

\section{Funding}

Paul Brogan acknowledges support from Great Ormond Street Hospital Children's Charity. All research at Great Ormond Street Hospital NHS Foundation Trust and UCL Great Ormond Street Institute of Child Health is made possible by the NIHR Great Ormond Street Hospital Biomedical Research Centre. The views expressed are those of the author(s) and not necessarily those of the NHS, the NIHR or the Department of Health. No other funding was obtained for this study.

\section{Availability of data and materials}

Availability of data and material: this report contains anonymised data and material collected from retrospective analyses of medical records stored as part of routine clinical care to preserve patient anonymity.

\section{Ethics approval and consent to participate}

Research approval was obtained from the Joint Research and Development office (R\&D Department) of GOSH. The R\&D reference number was $16 I R 47$. As per their recommendation, formal ethical approval was not required since this was a retrospective case note review with the presentation of anonymised data. Accordingly, patients and families did not have to sign a consent form to be included in the study.

\section{Consent for publication}

Not applicable (retrospective case notes review only).

\section{Competing interests}

Paul Brogan has received consultancy fees for Novartis, Roche, and SOBl; and institutional grants from Novartis, Roche, SOBI, and Novimmune. Helen J Lachmann has received consultancy fees for Novartis, Takeda and SOBI. Philip N Hawkins has received consultancy fees for Novartis, GSK, and Alnilam. None of the other authors had any disclosures.

\section{Author details}

${ }^{1}$ Infection Inflammation and Rheumatology Section, University College London Institute of Child Health, 30 Guilford St, London WC1N1EH, UK. 2Department of Paediatric Rheumatology, Great Ormond Street Hospital NHS Foundation Trust, London, UK. ${ }^{3}$ University College London National Amyloidosis Centre, London, UK.
Received: 8 February 2019 Accepted: 31 May 2019

Published online: 08 July 2019

\section{References}

1. Hoffman HM, Mueller $\mathrm{J}$, Broide DH, Wanderer AA, Kolodner RD. Mutation of a new gene encoding a putative pyrin-like protein causes familial cold autoinflammatory syndrome and muckle-Wells syndrome. Nat Genet. 2001; 29(3):301-5.

2. Aksentijevich I, Putnam CD, Remmers EF, Mueller JL, Le J, Kolodner RD, Moak Z, Chuang M, Austin F, Goldbach-Mansky R, Hoffman HM, Kastner DL. The clinical continuum of cryopyrinopathies: novel CIAS1 mutations in north American patients and a new cryopyrin model. Arthritis Rheum. 2007:56(4):1273-85.

3. Russo RA, Melo-Gomes S, Lachmann HJ, Wynne K, Rajput K, Eleftheriou D, Edelsten C, Hawkins PN, Brogan PA. Efficacy and safety of canakinumab therapy in paediatric patients with cryopyrin-associated periodic syndrome: a single-Centre, real-world experience. Rheumatology (Oxford). 2014;53(4): $665-70$.

4. ter Haar NM, Oswald M, Jeyaratnam J, Anton J, Barron KS, Brogan PA, Cantarini L, Galeotti C, Grateau G, Hentgen V, Hofer M, Kallinich T, Kone-Paut I, Lachmann HJ, Ozdogan H, Ozen S, Russo R, Simon A, Uziel Y, Wouters C, Feldman BM, Vastert SJ, Wulffraat NM, Benseler SM, Frenkel J, Gattorno M, Kuemmerle-Deschner JB. Recommendations for the management of autoinflammatory diseases. Ann Rheum Dis. 2015;74(9):1636-44.

5. Lachmann HJ, Kone-Paut I, Kuemmerle-Deschner JB, Leslie KS, Hachulla E, Quartier P, Gitton X, Widmer A, Patel N, Hawkins PN. Canakinumab in CSG. Use of canakinumab in the cryopyrin-associated periodic syndrome. N Engl Med. 2009:360(23):2416-25.

6. Kuemmerle-Deschner JB, Ozen S, Tyrrell PN, Kone-Paut I, Goldbach-Mansky R, Lachmann H, Blank N, Hoffman HM, Weissbarth-Riedel E, Hugle B, Kallinich T, Gattorno M, Gul A, Ter Haar N, Oswald M, Dedeoglu F, Cantarini L, Benseler SM. Diagnostic criteria for cryopyrin-associated periodic syndrome (CAPS). Ann Rheum Dis. 2017:76(6):942-7.

\section{Publisher's Note}

Springer Nature remains neutral with regard to jurisdictional claims in published maps and institutional affiliations.
Ready to submit your research? Choose BMC and benefit from:

- fast, convenient online submission

- thorough peer review by experienced researchers in your field

- rapid publication on acceptance

- support for research data, including large and complex data types

- gold Open Access which fosters wider collaboration and increased citations

- maximum visibility for your research: over $100 \mathrm{M}$ website views per year

At $\mathrm{BMC}$, research is always in progress.

Learn more biomedcentral.com/submission 